\title{
NMR Studies of a Model Antimicrobial Peptide in the Micelles of SDS, Dodecylphosphocholine, or Dioctanoylphosphatidylglycerol
}

\author{
Guangshun Wang* \\ The Structure-Fun Laboratory, Eppley Institute for Research in Cancer and Allied Diseases, University of Nebraska
Medical Center, 986805 Nebraska Medical Center, Omaha, NE 68198-6805, USA
}

Abstract: NMR analysis of GI-20 in three micelles revealed that arginine side-chain $\varepsilon^{\mathrm{N}}$ resonances are well resolved in dioctanoylphosphatidylglycerol (D8PG), allowing observations of peptide-lipid interactions. Structural differences in SDS and D8PG underscore caution with the use of SDS. Peptide chemical shifts are proposed to be a useful indicator of micelle environments.

Keywords: Antimicrobial peptides, chemical shifts, DPC, D8PG, lipid micelles, membrane proteins, NMR, SDS, spectral resolution, structural biology.

\section{INTRODUCTION}

Antimicrobial peptides are host defense molecules of innate immunity. As effector molecules, they are capable of rapidly eliminating invading pathogenic microbes such as bacteria. As signaling molecules, they may trigger adaptive immune responses in vertebrates to initiate a second round of attacks on invading microbes. Cumulative evidence suggests that it is more difficult for bacteria to develop drug resistance against antimicrobial peptides than traditional antibiotics. As a result, there is growing interest worldwide to develop novel antimicrobial agents based on natural antimicrobial peptides [1-4]. The 2007 version of the antimicrobial peptide database (APD) holds more than 780 entries identified from a variety of species, ranging from bacteria, fungi, amphibians, insects, to mammals, including humans [5]. While some of the peptides in the database have a wide-spectrum of toxicity to bacteria, viruses, fungi, and cancer cells, others show selective toxicity on bacteria with little effects on mammalian cells. It is the latter class of antimicrobial peptides that is of great therapeutic importance. The selectivity of these peptides is attributed to the fundamental differences in the membrane composition and structure of bacterial and human cells [6]. While human cellular membranes are composed primarily of zwitterionic phosphatidylcholines, bacterial membranes are rich in anionic phosphatidylglycerols (PGs). Electrostatic interactions between anionic lipids in bacterial membranes and cationic peptides may be the key for cellular selectivity of antimicrobial peptides.

Antimicrobial peptides are also good models for structural studies of membrane proteins, one of the most challenging problems of modern structural biology [6-11]. Threedimensional structures of antimicrobial peptides have greatly improved our understanding of their mechanisms of action. Structures of representative peptides can be viewed at the antimicrobial peptide database website http://aps.unmc.edu/ AP/main.html [5]. Because of the complexity of the bacterial

*Address correspondence to this author at the Eppley Institute, Room ECI3018, University of Nebraska Medical Center, 986805 Nebraska Medical Center, Omaha, NE 68198-6805, USA; Tel: (402) 559-4176; Fax: (402) 559-4651; E-mail: gwang@unmc.edu membranes, such peptide structures were mainly determined by solution NMR using membrane-mimetic models such as organic solvents or detergent micelles. Both deuterated SDS and DPC micelles are widely employed [7-11]. They share the same 12-carbon alkyl chain (Fig. 1A \& B). While negatively charged SDS is used to mimic bacterial membranes, DPC, with an identical head group to phosphatidylcholines in human cells, is utilized to mimic human cellular membranes. Because the major anionic lipids in bacterial membranes are PGs, structural studies in such a membranemimetic model should provide long-desired insight into peptide-PG interactions at atomic resolution. As a consequence, my laboratory has been exploring the utilization of PGs as an alternative bacterial membrane model for structural studies of bacterial membrane-targeting peptides such as antimicrobial peptides [12-18]. Our major findings are summarized here: (1) The chain length of PGs, ranging from six to 10 , had little effect on the conformation of the bacterial membrane anchor peptide [13]. (2) PG micelles dominate the translational diffusion coefficients of the peptide-lipid complex and the complex size increases with lipid chain length [14]. (3) The spectral line width of the micelle-bound peptides increases with the increase in lipid chain length. Taken together factors such as peptide structures, peptide-lipid ratios, and spectral line widths, D8PG (Fig. 1C) was chosen as a model [13]. (4) Aromatic rings of phenylalanines are useful probes for the penetration depth of the peptide into the D8PG micelle [15,17]. (5) Intermolecular NOE cross peaks were also observed between interfacial cationic arginine side chains and anionic D8PG [18]. (6) Similar structures were observed for short peptides ( 13 residues) in SDS and D8PG $[12,15,16]$. These short peptides include the bacterial membrane anchor from E. coli, antibacterial and anti-cancer peptide aurein 1.2 from Australian frogs, and an aurein 1.2 ana$\log$ derived from the only cathelicidin LL-37 from humans. Because of the limited cases, the structural similarities observed in SDS and D8PG should not be generalized. Indeed, we report here that SDS may induce unwanted structural artifacts. The model peptide (GI-20) used in this study corresponds to residues 13-32 of human LL-37 with the positions between residues I13 and G14 swapped [18], leading to the final peptide sequence GIKEFKRIVQRIKDFLRNLV. The 
residue numbering of LL-37 is adopted with the first residue as residue 13. Our results obtained in SDS, DPC, and D8PG micelles allow us to appreciate, for the first time, the uniqueness of D8PG in providing an excellent spectral window for observing peptide-PG interactions. Our studies also suggest that peptide chemical shifts may be utilized as useful probes for micellar environments.

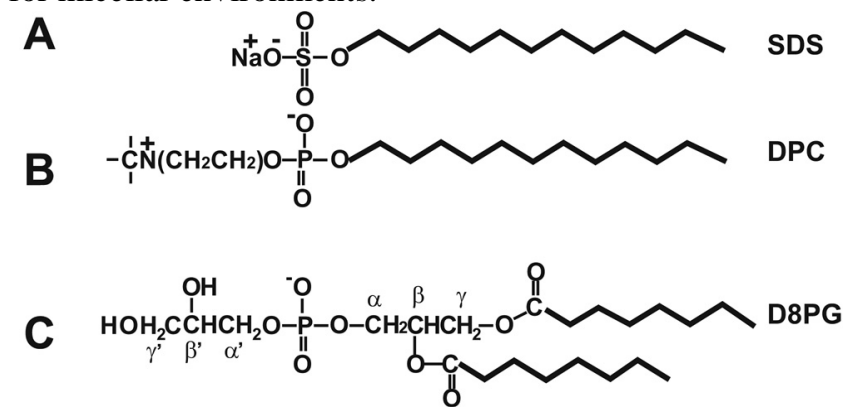

Fig. (1). Chemical structure of SDS (A), DPC (B), and D8PG (C).

\section{MATERIALS AND METHODOLOGY}

GI-20 with C-terminal amidation (> 95\% pure) was synthesized and purified by Genemed Synthesis (San Francisco, CA). Deuterated SDS and DPC were purchased from Cambridge Isotope Laboratories (Andover, MA). Protonated D8PG (> 98\%) was purchased from Avanti Polar Lipids (Alabaster, AL). Chloroform was removed from D8PG under a stream of nitrogen gas followed by evaporation under vacuum overnight.

\section{NMR Spectroscopy}

Three NMR samples were prepared by mixing the peptide $(2 \mathrm{mM})$ with a particular lipid or detergent at molar ratios of 1:40, 1:60, and 1:5 for SDS, DPC, and D8PG, respectively. Such ratios were found to be sufficient to stabilize the structure of the peptide. In addition, each sample contains $10 \% \mathrm{D}_{2} \mathrm{O}$ as the field-locking signal. The $\mathrm{pH}$ of each sample was adjusted to 5.4 by using microliter aliquots of $\mathrm{HCl}$ or $\mathrm{NaOH}$ solution and measured directly in the 5-mm NMR tube with a micro-pH electrode (Wilmad-Labglass).

NMR Data were recorded on a Varian INOVA 600-MHz NMR spectrometer equipped with a triple-resonance cryogenic probe with $\mathrm{z}$-axis gradient capability. For either SDSor DPC-containing peptide sample, TOCSY [19-21], DQFCOSY [22], and NOESY [23] spectra were collected in the States-TPPI mode. Typically, the mixing time was $75 \mathrm{~ms}$ for TOCSY and $100 \mathrm{~ms}$ for NOESY. For the D8PG-containing peptide sample, a 2D NOESY spectrum (75 ms) was recorded with 440 increments (64 scans each) in t1 and $2 \mathrm{~K}$ complex points in 2 time domains, using a spectral width of $8500 \mathrm{~Hz}$ in both dimensions with the ${ }^{1} \mathrm{H}$ carrier on water. In TOCSY and NOESY experiments, the water signal was suppressed by WATERGATE [24] and in the DQF-COSY experiment water was suppressed by presaturation during spin relaxation. For all the samples, a natural abundance $\left({ }^{1} \mathrm{H},{ }^{15} \mathrm{~N}\right)$ HSQC spectrum [25] was collected, typically with 30 increments (512 scans each) in the indirect dimension (60 increments in D8PG). The carrier was placed at the water resonance $(4.7 \mathrm{ppm})$ in the proton dimension (spectral width $8500 \mathrm{~Hz}$ ) and at $118.2 \mathrm{ppm}$ in the nitrogen dimension (spectral width $2200 \mathrm{~Hz}$ ). A natural abundance HSQC spectrum correlating aromatic ${ }^{13} \mathrm{C}$ (spectral width $3000 \mathrm{~Hz}, 30$ incre- ments) and ${ }^{1} \mathrm{H}$ (spectral width $8500 \mathrm{~Hz}$ ) was collected. In deuterated micelles, another HSQC for aliphatic ${ }^{13} \mathrm{C}$ and ${ }^{1} \mathrm{H}$ was also recorded to extract natural abundance ${ }^{13} \mathrm{C} \alpha$ and ${ }^{13} \mathrm{C} \beta$ chemical shifts.

NMR data were processed on a Silicon Graphics Octane workstation (SGI) using the NMRPipe software [26]. The time domain data were apodized by a $63^{\circ}$ shifted squared sine-bell window function in both dimensions, and zerofilled prior to Fourier transformation. For natural abundance HSQC, the data in the indirect dimension was doubled by linear prediction prior to Fourier transformation. Since anionic DSS is known to interact with cationic peptides [12], proton chemical shifts of the peptides were referenced to external DSS at $0.00 \mathrm{ppm}$. NMR data were analyzed with NMRDraw in the NMRPipe package [26] and PIPP [27].

\section{Three-Dimensional Structural Calculations}

The structure of GI-20 in complex with D8PG was calculated based on both distance and angle restraints by using the simulated annealing protocol in Xplor-NIH [28] as described previously [18]. The structures of the peptide in DPC and SDS were determined in the same manner. In brief, the distance restraints were obtained by classifying the NOE crosspeak volumes into strong (1.8-2.8 $\AA$ ), medium (1.8-3.8 $\AA$ ), weak (1.8-5.0 $\AA$ ), and very weak (1.8-6.0 $⿱$ ) ranges. The distances were calibrated on the basis of the typical NOE patterns in an $\alpha$ helix [7]. The backbone $\phi$ and $\psi$ angles of GI-20 in SDS and DPC micelles were predicted by TALOS [29] based on ${ }^{1} \mathrm{H} \alpha,{ }^{15} \mathrm{~N},{ }^{13} \mathrm{C} \alpha$, and ${ }^{13} \mathrm{C} \beta$ chemical shifts. A broader range $\left( \pm 20^{\circ}\right.$ ) than predicted (usually $< \pm 10^{\circ}$ ) was allowed for each angle in structural calculations. A covalent peptide structure with random $\phi, \psi$, and $\chi$ angles but trans planar peptide bonds was used as a starting structure. The peptide structural template was amidated at the C-terminus using X-PLOR. In total, 100 structures were calculated and an ensemble of 20 structures with the lowest total energy was chosen for structural analysis. This accepted structural ensemble also satisfies the following criteria: no NOE violations greater than $0.50 \AA$, RMSD for bond deviations from ideality less than $0.01 \AA$, and RMSD for angle deviations from ideality less than $5^{\circ}$. Structures were viewed and analyzed by MOLMOL [30] and PROCHECK [31].

\section{RESULTS AND DISCUSSION}

\section{D8PG Is Unique in Providing an Ideal Window for Detecting Arg-PG Interactions}

Fig. (2) presents select spectral regions of GI-20 in SDS, DPC, and D8PG micelles. The backbone amide region of the peptide covers a broader spectral range in DPC (9.2-7.5 ppm) or D8PG (9.1-7.5 ppm) than in SDS (8.6-7.7 ppm). A wider spectral region was also observed previously in DHPG, D8PG, or D10PG for the amide protons of the bacterial membrane anchor than in SDS [12]. The NMR signals of GI-20 in the three micelles were assigned by using the standard procedure [7]. In SDS, the aromatic and arginine $\varepsilon \mathrm{H}^{\mathrm{N}}$ resonances overlap (Fig. 2A). In DPC, the same arginine resonances overlap with the backbone amide region (Fig. 2B). In D8PG, however, the arginine $\varepsilon \mathrm{H}^{\mathrm{N}}$ chemical shifts are located between the backbone amide and aromatic proton resonances of the peptide (Fig. 2C). Because the PG head group is biologically more relevant, the best spectral resolu- 
tion observed in this spectral region for GI-20 in D8PG is remarkable. Since the interactions between peptide cationic residues and anionic lipids are believed to be critical for selective bacterial membrane targeting, the ideal location of the arginine side chain resonances in the spectrum (Fig. 2C) facilitates the observation of the Arg-PG interaction by NMR. Indeed, we previously demonstrated that both the aromatic rings and arginine side chains of antimicrobial peptides showed intermolecular NOE cross peaks with D8PG $[17,18]$. Thus, the current NMR study of the peptide in SDS, DPC, and D8PG micelles enabled us to appreciate the uniqueness of D8PG in terms of NMR spectra for the first time (Fig. 2).



Fig. (2). Part of the NOESY spectra of peptide GI-20 in different micelles. Panel (A) is the peptide inSDS micelles at a peptide/SDS ratio of 1:40, pH 5.4, and $25^{\circ} \mathrm{C}$, panel (B) is the peptide in $\mathrm{DPC}$ at a peptide/DPC ratio of $1: 60, \mathrm{pH} 5.4$, and $25^{\circ} \mathrm{C}$, and panel (C) is the peptide in D8PG at a peptide/lipid ratio of 1:5, pH 5.4, and $30^{\circ} \mathrm{C}$. The labile $\varepsilon \mathrm{H}^{\mathrm{N}}$ chemical shifts of the three arginine side chains of the peptide measured in D8PG at $25^{\circ} \mathrm{C}$ to $30^{\circ} \mathrm{C}$ differ by only $0.02 \mathrm{ppm}$. The signals for the backbone amide protons of $\mathrm{N} 30$, L31, and V32 (black), aromatic side chains of F17 and F27 (green), and arginine $\varepsilon \mathrm{H}^{\mathrm{N}}$ side chains (R19s, R23s, and R29s, in red) are labeled in color. In panel (B), R19 adjacent to R29 was too weak to see in this plot. Note the signal overlaps between the aromatic and arginine side chains in SDS and the overlaps between peptide backbone amide and arginine side chain protons in DPC.

\section{Three-Dimensional Structures of GI-20 in SDS, DPC, and D8PG Micelles}

To evaluate the impact of different micelles on the structure of the peptide, we also determined the structure of GI-20 in SDS (Fig. 3A) and DPC (Fig. 3C). The 3D structure of
GI-20 in D8PG (Fig. 3B) was reported previously [18]. Select statistical data are listed in Table 1. Clearly, the helical region of GI-20 in DPC is identical to that in D8PG. However, the structure of GI-20 in SDS differs at the N-terminus, which is not $\alpha$-helical. Such a structural difference is determined by the NOE differences of the peptide in SDS compared to the two phosphate-containing micelles. In particular, the $\mathrm{H \alpha}_{\mathrm{i}}-\mathrm{HN}_{\mathrm{i}+3}$ and $\mathrm{H} \alpha_{\mathrm{i}}-\mathrm{HN}_{\mathrm{i}+4}$ types of NOE connectivities of the peptide are more homogeneous in DPC or D8PG than in SDS (see supporting Fig. S1).

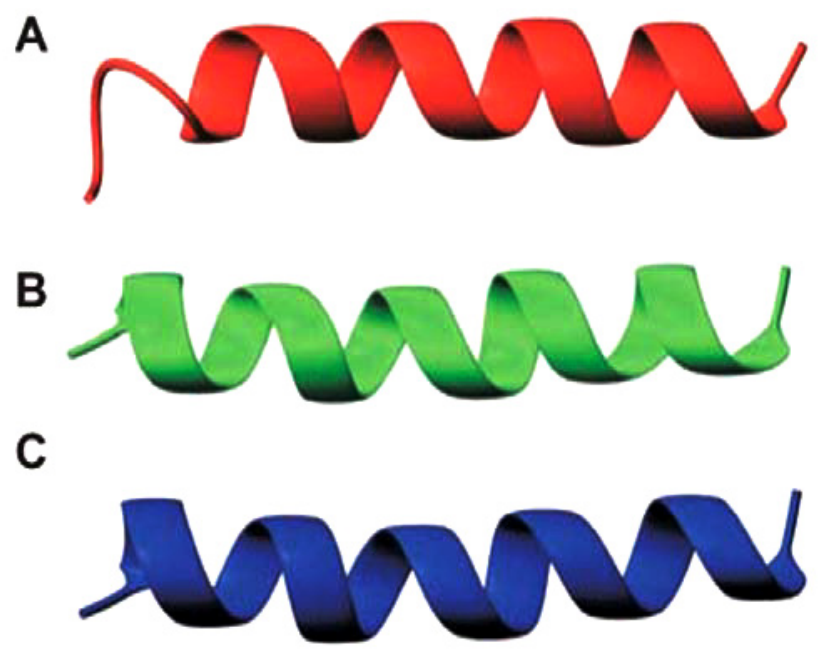

Fig. (3). Ribbon diagrams of representative structures of GI-20 in different micelles. The structures were determined in the presence of SDS (A), D8PG (B), and DPC (C), respectively. The images were made using MOLMOL [30].

To find additional support for the subtle structure difference for GI-20 in SDS and D8PG, we also compared the backbone chemical shift plots of the peptide in three micelles, since chemical shifts are well known to contain structural information [7,34]. The backbone amide proton plots superimpose nicely in D8PG and DPC (Fig. 4A). The plot in SDS is also similar except for the N-terminus (residues 1318). Such a difference in amide proton chemical shifts may, or may not, reflect structural difference. However, the $\mathrm{H} \alpha$ chemical shifts in the same peptide region also deviate in SDS (Fig. 4B), suggesting conformational change. Based on the average $\mathrm{H} \alpha$ secondary shift of the peptide divided by 0.4 [34], the helicities of GI-20 in the three micelles were calculated. While the helicity was $82 \%$ in SDS, the values in D8PG and DPC were higher at $93 \%$ and $97 \%$, respectively, consistent with the more helical structure determined in the latter two micelles with native lipid head groups (Fig. 3).

To understand the structural difference, we also examined the 3D structures of the peptide, especially in the $\mathrm{N}$ terminal region where differences occurred. An acidic side chain (Glu16) was found to be located in the interfacial region of the amphipathic structure [18]. Thus, it is likely that stronger electrostatic repulsions between this acidic Glu16 side chain and the negatively charged SDS head group distorted the helical structure locally. The similar electrostatic repulsions are expected to be reduced in D8PG and the weakest in DPC. This is because sulfate is related to a strong acid while phosphate is related to an intermediate acid. This offers one possible interpretation for the above helicity order (DPC > D8PG > SDS) observed in the three micelles. 
Table 1. Structural Statistics for GI-20 Bound to SDS, DPC, or D8PG Micelles

\begin{tabular}{|c|c|c|c|}
\hline NOE restraints & SDS & DPC & D8PG [18] \\
\hline Intra-residue & 78 & 83 & 75 \\
\hline Short range & 44 & 70 & 44 \\
\hline Total & 187 & 220 & 182 \\
\hline \multicolumn{4}{|l|}{ RMSD for superimposing residues $14-31(\AA)$} \\
\hline Backbone & 0.51 & 0.49 & 0.55 \\
\hline Heavy atoms & 1.21 & 1.18 & 1.31 \\
\hline In the additional allowed region & $0 \%$ & $0 \%$ & $5.6 \%$ \\
\hline Dominate helical region in the structural ensemble & $17-31$ & $14-31$ & $14-31$ \\
\hline
\end{tabular}

SDS and DPC have been in use for many years and it is not surprising that subtle structural differences were observed previously in some cases [e.g. 32, 35, 36]. Nevertheless, the interpretation of those structural differences might be more complex since the head group of SDS differs from that of DPC not only chemically but also in charge (Fig. 1). Our structural comparison of GI-20 in SDS and D8PG here enabled us to narrow down the possibilities to the sulfate group rather than the negative charge. Thus, it is a good idea to be cautious with the use of strong ionic detergents such as SDS as a membrane-mimetic model for structural studies of membrane proteins. Because we observed a good correlation between backbone 3D structures and $\mathrm{H} \alpha$ chemical shifts of the peptide, comparison of $\mathrm{H} \alpha$ chemical shifts should be a useful indicator for potential structural change in SDS relative to those in phospholipids.

\section{Peptide Chemical Shifts as a Useful Probe for the Types of Micelles}

Since peptide side chains are expected to directly associate with the micelles, we also plotted the chemical shifts for hydrophobic aromatic F17 and F27 (Fig. 4C) as well as the $\varepsilon \mathrm{H}^{\mathrm{N}}$ chemical shifts of R19, R23, and R29 of GI-20 (Fig. 4D). The chemical shifts of the hydrophobic F17 and F27 varied only slightly, suggesting similar hydrophobic environments in all three micelles. However, significant variations were observed for cationic side chains. The magnitude of arginine $\varepsilon \mathrm{H}^{\mathrm{N}}$ chemical shift is in the following order: DPC $>$ D8PG > SDS (Fig. 4D). In agreement, an earlier study found that the arginine $\varepsilon \mathrm{H}^{\mathrm{N}}$ resonances of human apolipoprotein A-I peptides were on average $0.43 \mathrm{ppm}$ larger in zwitterionic DPC than in anionic SDS [32]. In contrast, chemical shift data for lysine $\mathrm{NH}_{3}{ }^{+}$side chains are rare in the literature, probably due to fast exchange on the NMR time scale. However, the chemical shifts of the lysine side chain $\mathrm{NH}_{3}{ }^{+}$
A

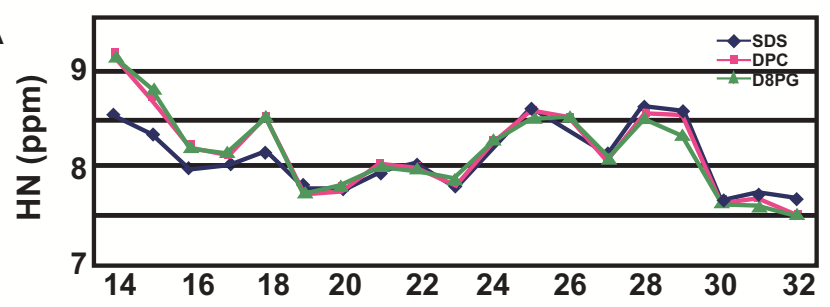

B

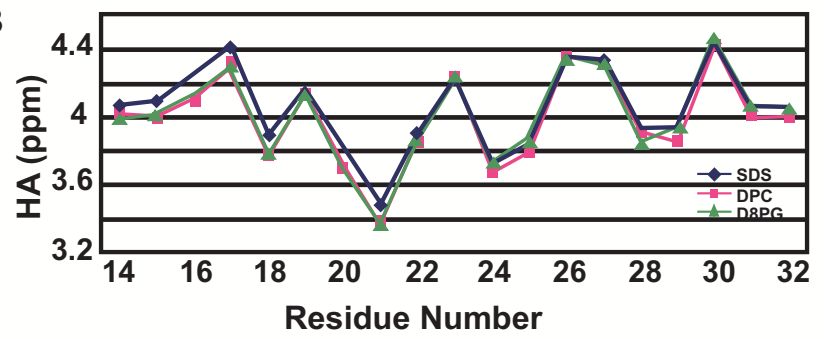

C

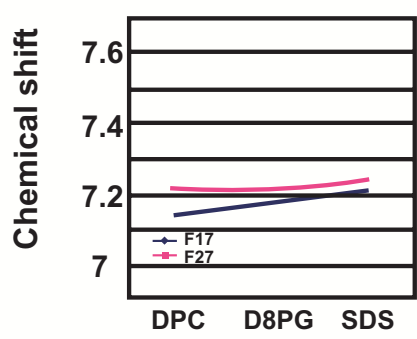

D

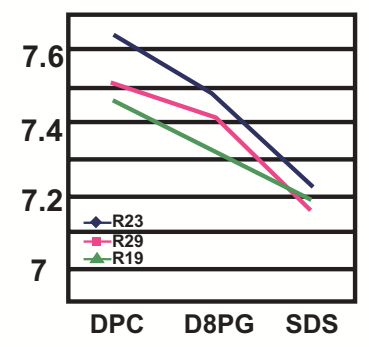

Fig. (4). Chemical shift plots for the backbone $\alpha$-protons (A), amide protons $(\mathbf{B})$, aromatic $\mathrm{H} \delta$ resonances of hydrophobic side chains of phenylalanines $\mathrm{F} 17$ and $\mathrm{F} 27(\mathbf{C})$, and $\varepsilon \mathrm{H}^{\mathrm{N}}$ resonances of cationic arginine side chains (D) of the peptide (GI-20) in SDS, DPC, and D8PG micelles. The lines are color-labeled in each panel. 
protons of a human apolipoprotein A-I-mimicking peptide [33] were found to be smaller in SDS than in DPC. Thus, the upfield shifts of cationic side chain resonances in SDS may be general. Importantly, the order of chemical shifts of arginine side chain in SDS, D8PG, and DPC (Fig. 4) appear to be correlated with the strength of the detergent head groups. The stronger the head group, the smaller the chemical shifts. Likewise, we previously observed that the magnitude of backbone amide chemical shifts for the $\mathrm{N}$-terminal residues (L2 and F3) of the bacterial membrane anchor is PGs > alkyl sulfonates $>$ SDS (see supporting Fig. S2) [14]. The shifts for L2 and F3 in sulfonates are nearly identical, indicating the hydrophobic chain length or chain branching had little effect. Therefore, these chemical shift orders appear to correlate with the strength of ionic interactions between the peptide and detergents. A detergent head group is regarded as strong if the corresponding acid is strong (as measured by pKa).

\section{CONCLUSION}

PGs are not only critical for selective bacterial membrane targeting by cationic antimicrobial peptides [6], they are also known to be essential for other molecular events in bacteria such as signal transduction [37] and normal functioning of voltage-dependent $\mathrm{K}^{+}$channel KvAP [38]. Our comparative NMR study of a model membrane peptide in SDS, DPC, and D8PG enabled us to appreciate the uniqueness of the PG head group for the first time. This is reflected in the intermediate values in terms of both chemical shift (Fig. 4D) and helicity of the peptide. It is remarkable to find that D8PG, as a biologically more relevant model, also yielded the bestresolved NMR spectra (Fig. 2). In particular, the location of the arginine $\varepsilon \mathrm{H}^{\mathrm{N}}$ side chain resonances between the backbone amide and the aromatic resonances opened an excellent spectral window for observing the interactions between cationic peptides and anionic PGs. Indeed, intermolecular NOE cross peaks have been detected between interfacial arginines of the GI-20 and D8PG, providing direct evidence for ArgPG interactions by solution NMR [18]. The magnitude of the chemical shifts of the arginine side chains in SDS, D8PG, and DPC suggests that chemical shifts are useful reporters of the micelle environments. The similarities of DPC and D8PG in terms of NMR spectra and peptide backbone structures may result from the common phosphate moiety in the lipid head groups (Fig. 1). This study also allowed us to better evaluate the impact of SDS on the structures of GI-20. The structural differences observed in SDS and D8PG indicate that SDS may cause structural artifacts probably due to stronger-than-desired electrostatic interactions. Our results support the use of DPC or D8PG with native lipid head groups as membrane-mimetic models. Indeed, based on the quality of HSQC spectra, the Girvin group found that singlechain PGs (lysoPGs) are useful for several membrane proteins with 1-4 transmembrane helices [39]. In addition, Cross and colleagues found that DPC gave useful NMR spectra for most of the membrane proteins investigated in their study [40]. Taken together, we propose that DPC and PGs be utilized as the first choice as membrane-mimetic models for structural studies of membrane proteins by NMR. If difficulty is met, other membrane-mimetic models, including a mixture of lipids containing DPC or PGs, may be pursued [41].
ABBREVIATIONS

$\begin{array}{ll}\text { NMR } & =\text { Nuclear magnetic resonance } \\ \text { DHPG } & =\text { Dihexanoylphosphatidylglycerol } \\ \text { D8PG } & =\text { Dioctanoylphosphatidylglycerol } \\ \text { D10PG } & =\text { Didecanoylphosphatidylglycerol } \\ \text { DPC } & =\text { Dodecylphosphocholine } \\ \text { DQF-COSY } & =\text { Double-quantum filtered correlation spec- } \\ & \text { troscopy } \\ \text { DSS } & =2,2 \text {-dimethyl-silapentane-5-sulfonate so- } \\ & \text { dium salt } \\ \text { HSQC } & =\text { Heteronuclear single quantum coherence } \\ \text { NOE } & =\text { Nuclear Overhauser effect } \\ \text { NOESY } & =\text { Nuclear Overhauser enhancement spec- } \\ \text { PGs } & =\text { troscopy } \\ \text { ppm } & =\text { Parts per million } \\ \text { RMSD } & =\text { Root mean square deviation } \\ \text { SDS } & =\text { Sodium dodecylsulfate } \\ \text { TOCSY } & =\text { Total correlation spectroscopy }\end{array}$

\section{ACKNOWLEDGEMENTS}

Funding from the University of Nebraska Medical Center is appreciated. The NMR Core Facility is supported by the Eppley Cancer Center CORE grant from the National Cancer Institute (P30CA036727) as well as Nebraska Research Initiative (NRI). In particular, recent NRI funding (to GW) that allowed the purchase of a new 400-MHz NMR spectrometer is greatly acknowledged. The author thanks Ed Ezell for careful reading of the final version of the manuscript.

\section{REFERENCES}

[1] Boman HG. Peptide antibiotics and their role in innate immunity. Annu Rev Immunol 1995; 13: 61-92.

[2] Zasloff M. Antimicrobial peptides of multicellular organisms. Nature 2002; 415: 389-395.

[3] Sambhara S, Lehrer RI. The innate immune system: a repository for future drugs? Expert Rev. Anti Infect Ther 2007; 5: 1-5.

[4] Brown KL, Cosseau C, Gardy JL, Hancock RE. Complexities of targeting innate immunity to treat infection. Trends Immunol 2007; 28: 260-266.

[5] Wang Z, Wang G. APD: the antimicrobial peptide database. Nucleic Acids Res 2004; 32: D590-D592.

[6] Epand RM, Vogel HJ. Diversity of antimicrobial peptides and their mechanisms of action. Biochim Biophys Acta 1999; 1462: 11-28.

[7] Wüthrich K. NMR of Proteins and Nucleic Acids, 1986, Wiley, New York.

[8] Gesell J, Zaslof M, Opella SJ. Two-dimensional 1H NMR experiments show that the 23-residue magainin antibiotic peptide is an alpha-helix in dodecylphosphocholine micelles, sodium dodecylsulfate micelles, and trifluoroethanol/water solution. J Biomol NMR 1997; 9: 127-135.

[9] Rozek A, Friedrich CL, Hancock RE. Structure of the bovine antimicrobial peptide indolicidin bound to dodecylphosphocholine and sodium dodecyl sulfate micelles. Biochemistry 2000; 39: 15765-15774.

[10] Wang G. Structural biology of antimicrobial peptides by NMR spectroscopy. Curr Org Chem 2006; 10: 569-581.

[11] Haney EF, Lan F, Vogel HJ. Solution structures and model membrane interactions of lactoferrampin, an antimicrobial peptide derived from bovine lactoferrin. Biochim Biophys Acta 2007; 1768: 2355-2364. 
[12] Wang G, Keifer, PA, Peterkofsky A. Solution structure of the Nterminal amphitropic domain of Escherichia coli glucose-specific enzyme IIA in membrane-mimetic micelles. Protein Sci 2003; 12: 1087-1096.

[13] Wang G, Keifer PA, Peterkofsky A. Short-chain diacyl phosphatidylglycerols: which one to choose for the NMR structural determination of a membrane-associated peptide from Escherichia coli? Spectroscopy 2004; 18: 257-264.

[14] Keifer PA, Peterkofsky A, Wang G. Effects of detergent alkyl chain length and chemical structure on the properties of a micellebound bacterial membrane targeting peptide. Anal Biochem 2004; 331: 33-39.

[15] Wang G, Li Y, Li X. Correlation of three-dimensional structures with the antibacterial activity of a group of peptides designed based on a nontoxic bacterial membrane anchor. J Biol Chem 2005; 280: 5803-5811.

[16] Li X, Li Y, Han H, Miller DW, Wang G. Solution structures of human LL-37 fragments and NMR-based identification of a minimal membrane-targeting antimicrobial and anticancer region. J Am Chem Soc 2006; 128: 5776-5785.

[17] Li X, Li Y, Peterkofsky A, Wang G. NMR studies of aurein 1.2 analogs. Biochim Biophys Acta 2006; 1758: 1203-1214.

[18] Wang G. Determination of solution structure and lipid micelle location of an engineered membrane peptide by using one NMR experiment and one sample. Biochim Biophys Acta 2007; 1768: 3271-3281.

[19] Braunschweiler L, Ernst, RR. Coherence transfer by isotropic mixing: application to proton correlation spectroscopy. J Magn Reson 1983; 53: 521-528.

[20] Bax A, Davis DG. MLEV-17 based two-dimensional homonuclear magnetization transfer spectroscopy. J Magn Reson 1985; 65: 355360 .

[21] Griesinger C, Otting G, Wüthrich K, Ernst RR. Clean TOCSY for proton spin system identification in macromolecules. J Am Chem Soc 1988; 110: 7870-7872.

[22] Rance M, Sørensen OW, Bodenhausen G, Wagner G. Enrst RR, Wüthrich K. Improved spectral resolution in COSY ${ }^{1} \mathrm{H}$ NMR spectra of proteins via double quantum filtering. Biochem Biophys Res Commun 1983; 117: 479-485.

[23] Jeener J, Meier BH, Bachmann P, Ernst RR. Investigation of exchange processes by two-dimensional NMR spectroscopy. J Chem Phys 1979; 71: 4546-4553.

[24] Piotto M, Saudek V, Sklenar V. Gradient-tailored excitation for single-quantum NMR spectroscopy of aqueous solutions, J Biomol NMR 1992; 2: 661-665.

[25] Kay LE, Keifer PA, Saarinen T. Pure absorption gradient enhanced heteronuclear single quantum correlation spectroscopy with improved sensitivity. J Am Chem Soc 1992; 114: 10663-10665.

[26] Delaglio F, Grzesiek S, Vuister GW, Zhu G, Pfeifer J, Bax A. NMRPipe: a multidimensional spectral processing system based on UNIX pipes. J Biomol NMR 1995; 6: 277-293.
[27] Garrett DS, Powers R, Gronenborn AM, Clore GM. A common sense approach to peak picking two-, three- and four-dimensional spectra using automatic computer analysis of contour diagrams. J Magn Reson 1991; 95: 214-220.

[28] Schwieters CD, Kuszewski J, Tjandra N, Clore GM. The XplorNIH NMR molecular structure determination package. J Magn Reson 2002; 160: 65-73.

[29] Cornilescu G, Delaglio F, Bax A. Protein backbone angle restraints from searching a database for chemical shift and sequence homology. J Biomol NMR 1999; 13: 289-302.

[30] Koradi R, Billeter M, Wüthrich K. MOLMOL: a program for display and analysis of macromolecular structures. J Mol Graphics 1996; 14: 51-55.

[31] Laskowski RA, MacArthur MW, Moss DS, Thornton JM. J Appl Cryst. 1993; 26: 283-291.

[32] Wang G, Treleaven WD, Cushley RJ. Conformation of human serum apolipoprotein A-I(166-185) in the presence of sodium dodecyl sulfate or dodecylphosphocholine by $1 \mathrm{H}-\mathrm{NMR}$ and CD. Evidence for specific peptide-SDS interactions. Biochim Biophys Acta 1996; 1301: 174-184.

[33] Buchko GW, Treleaven WD, Dunne SJ, Tracey AS, Cushley RJ. Structural studies of a peptide activator of human lecithincholesterol acyltransferase. J Biol Chem 1996; 271: 3039-3045.

[34] Wishart DS, Sykes BD, Richards FM. Relationship between nuclear magnetic resonance chemical shift and protein secondary structure. J Mol Biol 1991; 222: 311-333.

[35] Neidigh JW, Andersen NH. Peptide conformational changes induced by tryptophan-phosphocholine interactions in a micelle. Biopolymers 2002; 65: 354-361.

[36] Hicks RP, Mones E, Kim H, Koser BW, Nichols DA, Bhattacharjee AK. Comparison of the conformation and electrostatic surface properties of magainin peptides bound to sodium dodecyl sulfate and dodecylphosphocholine micelles. Biopolymers 2003; 68: 459470.

[37] Wang G, Peterkofsky A, Clore GM. A novel membrane anchor function for the N-terminal amphipathic sequence of the signaltransducing protein IIAGlucose of the Escherichia coli phosphotransferase system. J Biol Chem 2000; 275: 39811-39814.

[38] Schmidt D, Jiang Q, MacKinnon R. Phospholipids and the origin of cationic gating charges in voltage sensors. Nature 2006; 444: 775 779.

[39] Krueger-Koplin RD, Sorgen PL, Krueger-Koplin ST, et al. An evaluation of detergents for NMR structural studies of membrane proteins. J Biomol NMR 2004; 28: 43-57.

[40] Page RC, Moore JD, Nguyen HB, et al. Comprehensive evaluation of solution nuclear magnetic resonance spectroscopy sample preparation for helical integral membrane proteins. J Struct Funct Genomics 2006; 7: 51-64.

[41] Wang G. NMR of membrane-associated peptides and proteins. Curr Protein Pept Sci 2008; In press. 
SDS G13114 K15E16 F17 K18 R19 120 V21 Q22 R23 124 K25 D26 F27 L28 R29 N30 L31 V32

$\mathrm{HNi-HNi+1}$ ? ? ? ? ? ? ? ? ? ? ? ? ? ? ? ? ?

$\alpha \mathrm{i}-\mathrm{HNi}+1$

$\alpha \mathrm{i}-\mathrm{HNi}+3$

(19

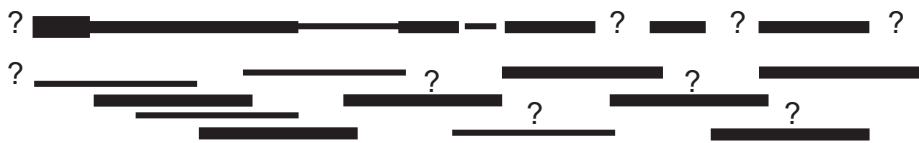

$\alpha \mathrm{i}-\mathrm{HNi}+4$

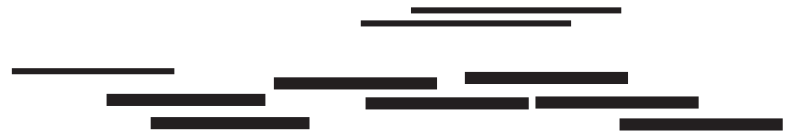

DPC G13 114 K15 E16 F17 K18 R19 120 V21 Q22 R23 124 K25 D26 F27 L28 R29 N30 L31 V32

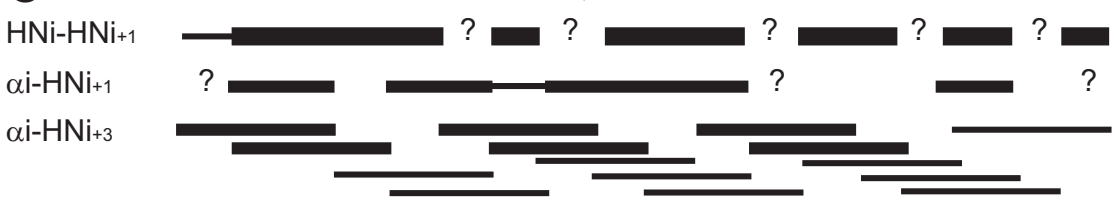

$\alpha \mathrm{i}-\mathrm{HNi}+4$

$\alpha \mathbf{i}-\beta \mathbf{i}+3$

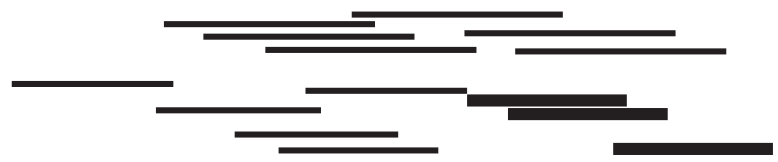

D8PG G13 114 K15E16 F17 K18 R19 120 V21 Q22 R23 124 K25 D26 F27 L28 R29 N30 L31 V32

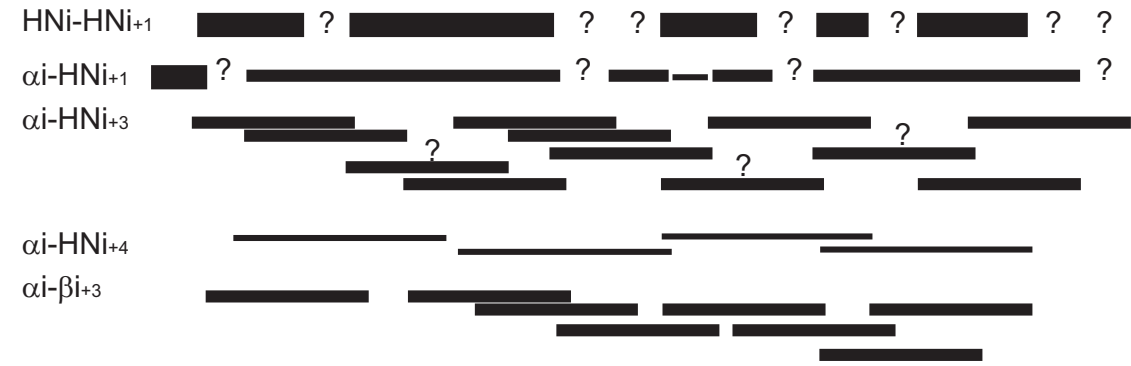

Fig. (S1). NOE connectivities of Gl-20 in SDS, DPC, and D8PG micelles. The thickness of the lines indicates NOE intensity (strong, medium, and weak). Question marks indicate uncertainty due to spectral overlap. Hydrophobic residues are in green and interfacial hydrophilic residues are in red.

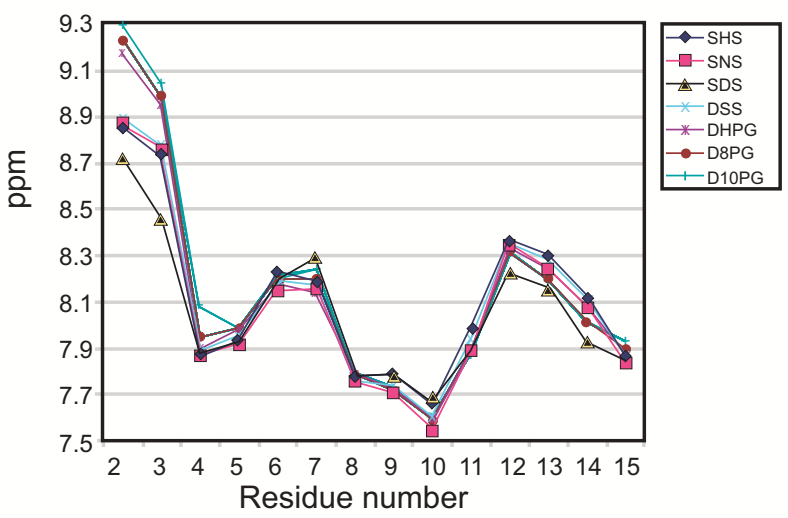

Fig. (S2). The backbone amide proton chemical shifts of the bacterial membrane anchor peptide in sodium hexanesulfonate (SHS), Sodium nonanesulfonate (SNS), SDS, DSS, DHPG, D8PG, and D10PG at pH 5.4 and $25^{\circ} \mathrm{C}$. Please refer to references [12] and [13] for additional experimental details. See the text for additional detergent abbreviations. 